\title{
Inventário das espécies de Lepturinae, Parandrinae e Prioninae (Insecta, Coleoptera, Cerambycidae) do Parque Nacional do Itatiaia, RJ, Brasil
}

\author{
Marcela Laura Monné ${ }^{1,2}$, Miguel Angel Monné $e^{1}$, Allan Carelli Aragão ${ }^{1}$, \\ Hingrid Yara Quintino ${ }^{I}$, Juan Pablo Botero ${ }^{I}$ \& Vanessa Souza Machado \\ ${ }^{1}$ Departamento de Entomologia, Museu Nacional, Universidade Federal do Rio de Janeiro - UFRJ, \\ Quinta da Boa Vista, São Cristóvão, CEP 20940-040, Rio de Janeiro, RJ, Brasil \\ ${ }^{2}$ Autor para correspondência: Marcela Laura Monné, e-mail: mlmonne@uol.com.br
}

MONNÉ, M.L., MONNÉ, M.A., ARAGÃO, A.C., QUINTINO, H.Y., BOTERO, J.P. \& MACHADO, V.S. Inventory of the Lepturinae, Parandrinae and Prioninae species (Insecta, Coleoptera, Cerambycidae) of the Parque Nacional do Itatiaia, RJ, Brazil. Biota Neotrop. 10(2): http://www.biotaneotropica.org.br/v10n2/ en/abstract?inventory+bn03610022010.

Abstract: A survey of the Lepturinae, Parandrinae and Prioninae species recorded in the Parque Nacional do Itatiaia, Rio de Janeiro State, Brazil, is presented. Five species of Lepturinae, eight of Parandrinae and 20 of Prioninae are registered, representing about $40 \%$ of the species that occur in the Atlantic Rainforest. Two new distribution records are registered to Prioninae species. Thirty one species are illustrated. A comparative table showing the respective number of genera and species of Lepturinae, Parandrinae and Prioninae that occur in the Neotropical Region, Atlantic Rainforest and Parque Nacional do Itatiaia is given.

Keywords: Cerambycidae, Coleoptera, inventory, Atlantic Rainforest.

MONNÉ, M.L., MONNÉ, M.A., ARAGÃO, A.C., QUINTINO, H.Y., BOTERO, J.P. \& MACHADO, V.S. Inventário das espécies de Lepturinae, Parandrinae e Prioninae (Insecta, Coleoptera, Cerambycidae) do Parque Nacional do Itatiaia, RJ, Brasil. Biota Neotrop. 10(2): http://www.biotaneotropica.org.br/v10n2/pt/a bstract?inventory+bn03610022010.

Resumo: É apresentado um levantamento das espécies de Lepturinae, Parandrinae e Prioninae que ocorrem no Parque Nacional do Itatiaia, Estado do Rio de Janeiro, Brasil. São registradas cinco espécies de Lepturinae, oito de Parandrinae e 20 de Prioninae, que representam $40 \%$ das espécies que ocorrem na Mata Atlântica. Novas ocorrências de distribuição são registradas para duas espécies de Prioninae. Trinta e uma espécies são ilustradas. É fornecida uma Tabela comparativa mostrando o número de gêneros e espécies de Lepturinae, Parandrinae e Prioninae que ocorrem na região Neotropical, na Mata Atlântica e no Parque Nacional do Itatiaia.

Palavras-chave: Cerambycidae, Coleoptera, inventário, Mata Atlântica. 


\section{Introdução}

Os Cerambycidae são besouros das mais diversas formas e tamanhos, podendo alcançar até $20 \mathrm{~cm}$, e constituem um grupo importante do ponto de vista florestal e agrícola já que as larvas são xilófagas, broqueando os troncos e galhos (Martins 1997). A família apresenta, na região Neotropical, sete subfamílias, cerca de 9.000 espécies e está dividida nas seguintes subfamílias: Aseminae, Cerambycinae, Lamiinae, Lepturinae, Parandrinae, Prioninae e Spondylidinae (Monné 2005a, b, 2006).

O Parque Nacional do Itatiaia (PNI) representa uma das mais importantes Unidades de Conservação da Mata Atlântica e, do ponto de vista da entomofauna, pouco se sabe sobre espécies de Coleoptera. Em Cerambycidae estão registradas cinco subfamílias: Cerambycinae, Lamiinae, Lepturinae, Parandrinae e Prioninae. Três inventários foram realizados no PNI: Zikán, J.F. \& Zikán, W. (1944) que trataram dos Cerambycidae que ocorrem no Itatiaia e na Mantiqueira onde registraram 718 espécies; Zajciw (1972) que tratou exclusivamente das espécies ocorrentes no Parque Nacional do Itatiaia, assinalando 588 espécies, sendo oito de Lepturinae, 10 de Parandrinae e 15 de Prioninae e Monné et al. (2009) que atualizaram o inventário de Cerambycinae no PNI, registrando 293 espécies.

Este trabalho visa dar continuidade ao projeto que vem sendo desenvolvido no PNI, sobre atualizar o inventário das espécies de Cerambycidae e que foi iniciado com a subfamília Cerambycinae (Monné et al. 2009). Objetiva-se atualizar os dados das espécies de Lepturinae, Parandrinae e Prioninae que ocorrem no parque com base em dados da literatura, da coleção de Cerambycidae do Museu Nacional, Universidade Federal do Rio de Janeiro e de coletas de campo; contribuir com dados de distribuição para as espécies e, eventualmente, novos registros. Fornecer, pela primeira vez, uma tabela, atualizada até fevereiro de 2010, com o número de gêneros e espécies de Lepturinae, Parandrinae e Prioninae que ocorrem na região Neotropical, na Mata Atlântica e no Parque Nacional do Itatiaia.

\section{Material e Métodos}

O Parque Nacional do Itatiaia situa-se geograficamente entre os paralelos $22^{\circ} 19^{\prime}$ e $22^{\circ} 45^{\prime} \mathrm{S}$ e os meridianos $44^{\circ} 15^{\prime}$ e $44^{\circ} 50^{\prime} \mathrm{O}$. Está localizado na divisa entre os estados do Rio de Janeiro, Minas Gerais e São Paulo, na Serra da Mantiqueira. Fica a Sudoeste do Estado do Rio de Janeiro, no município de Itatiaia, e a Sudoeste do Estado de Minas Gerais, abrangendo os Municípios de Itamonte, Alagoa e Bocaina de Minas (Brasil 2009).

Foram estudados os exemplares da coleção do Museu Nacional, Universidade Federal do Rio de Janeiro com registro para o Parque Nacional do Itatiaia e realizadas 10 coletas, entre setembro de 2007 e janeiro de 2010 (Licença de coleta do IBAMA n ${ }^{\circ}$ 16928-1); a literatura pertinente foi examinada com intuito de complementar o estudo. As coletas foram realizadas próximas à sede do parque, em altitudes de 700 a $1.250 \mathrm{~m}$; durante o dia utilizou-se guarda-chuva entomológico e rede entomológica e, durante a noite, pano branco com fonte luminosa próxima ao mesmo. O material coletado foi montado, etiquetado e depositado na coleção do Museu Nacional, Universidade Federal do Rio de Janeiro.

A lista é apresentada em ordem alfabética de subfamília, tribo, gênero e espécie e as referências sob cada espécie restringem-se à descrição original e à dos autores que citaram a espécie para o parque. O item distribuição, em cada espécie, foi obtido de Monné (2006), Monné, M.L. \& Monné, M.A. (2008) e Santos-Silva \& Martins (2010). O item material examinado é incluído quando ocorre um novo registro. As espécies obtidas nas coletas são destacadas com um asterisco ao lado do nome da espécie. Para a confecção da Tabela 1 foram utilizados os dados do catálogo de Monné (2006) e todos os trabalhos posteriores, até fevereiro de 2010, referentes a espécies ocorrentes na região neotropical. Siglas utilizadas no texto: MNRJ, Museu Nacional, Universidade Federal do Rio de Janeiro, Rio de Janeiro, Brasil; PNI, Parque Nacional do Itatiaia.

Tabela 1. Número de gêneros e espécies por tribo de Lepturinae, Parandrinae e Prioninae que ocorrem na região Neotropical, na Mata Atlântica e no Parque Nacional do Itatiaia.

Table 1. Number of genera and species by tribe of Lepturinae, Parandrinae and Prioninae that occur in the Neotropical Region, Atlantic Rainforest and Parque Nacional do Itatiaia.

\begin{tabular}{lccc}
\hline \multicolumn{1}{c}{$\begin{array}{c}\text { Subfamílias } \\
\text { tribos }\end{array}$} & $\begin{array}{c}\text { Gêneros /espécies } \\
\text { na região Neotropical }\end{array}$ & $\begin{array}{c}\text { Gêneros/espécies } \\
\text { na Mata Atlântica }\end{array}$ & $\begin{array}{c}\text { Gêneros/espécies } \\
\text { no Parque Nacional do Itatiaia }\end{array}$ \\
\hline Lepturinae & $12 / 62$ & & - \\
Necydalini & $39 / 174$ & $5 / 10$ & $2 / 5$ \\
Lepturini & $51 / 236$ & $3 / 13$ & $2 / 5$ \\
Total & & $8 / 23$ & - \\
Parandrinae & $1 / 1$ & & $2 / 8$ \\
Erichsonini & $3 / 41$ & - & $2 / 8$ \\
Parandrini & $4 / 42$ & $2 / 11$ & \\
Total & & $2 / 11$ & $1 / 1$ \\
Prioninae & $22 / 47$ & & \\
Anacolini & $10 / 33$ & $6 / 9$ & - \\
Callipogonini & $1 / 4$ & $6 / 10$ & $1 / 2$ \\
Calocomini & $5 / 17$ & $1 / 1$ & $2 / 2$ \\
Macrodontini & $18 / 62$ & $2 / 4$ & $2 / 2$ \\
Macrotomini & $10 / 28$ & $7 / 10$ & $3 / 6$ \\
Mallaspini & $11 / 26$ & $3 / 3$ & - \\
Meroscelisini & $8 / 58$ & $3 / 9$ & - \\
Prionini & $6 / 46$ & - & $14 / 20$ \\
Solenopterini & $91 / 321$ & - & \\
Total & & $28 / 46$ & \\
\hline
\end{tabular}




\section{Resultados}

São registradas cinco espécies de Lepturinae, oito de Parandrinae e 20 de Prioninae, que representam $40 \%$ das espécies que ocorrem na Mata Atlântica.

São registradas cinco espécies de Lepturinae distribuídas em dois gêneros. Não ocorreram novos registros de distribuição e não foram coletados exemplares. Das cinco espécies, duas apresentam ampla distribuição na América do Sul e três ocorrem apenas na Mata Atlântica.

Em Parandrinae registraram-se oito espécies, distribuídas em dois gêneros, e também não ocorreram novos registros de distribuição. Para a Mata Atlântica estão registradas 11 espécies e apenas três não ocorrem no PNI. Nas coletas foram obtidas duas espécies: Parandra (Parandra) glabra (Degeer, 1774) e Parandra (Parandra) minuscula Zikán, 1948.

Em Prioninae são registradas 20 espécies distribuídas em 14 gêneros e seis tribos. Dois novos registros de distribuição foram obtidos para o PNI: Meroscelisus apicalis White, 1853 e Quercivir dohrni Lameere, 1912. Para a Mata Atlântica estão registradas 46 espécies e cerca de $45 \%$ destas espécies ocorrem no PNI. Nas coletas realizadas no parque, foram obtidas sete espécies: Myzomorphus quadripunctatus (Gray, 1831), Chorenta reticulata (Dalman, 1817), Hephialtes ruber (Thunberg, 1822), Ancistrotus aduncus Buquet, 1853, Ancistrotus uncinatus (Klug, 1825), Mallodon spinibarbis (Linnaeus, 1758) e Mecosarthron buphagus Buquet, 1840.

Abaixo segue a lista das espécies de Lepturinae, Parandrinae e Prioninae que ocorrem no PNI.

\section{SUBFAMÍLIA LEPTURINAE}

Tribo Lepturini

1. Euryptera unilineatocollis Fuchs, 1956 (Figura 1)

Euryptera unilineatocollis Fuchs 1956: 567, Monné \& Monné 2008: 42.

Distribuição - Brasil (Espírito Santo a Santa Catarina), Paraguai, Argentina.

2. Strangalia flavocincta (Thomson, 1861) (Figura 2)

Ophistomis flavocinctus Thomson 1861a: 155.

Ophistomis latifasciata: Zajciw 1972: 47.

Ophiostomis latifasciata: Zikán \& Zikán 1944: 13.

Distribuição - Brasil (Bahia a São Paulo).

3. Strangalia fulvicornis (Bates, 1872) (Figura 3)

Ophistomis fulvicornis Bates 1872: 183.

Ophistomis variabilis: Zajciw 1972: 48.

Ophiostomis variabilis: Zikán \& Zikán 1944: 13.

Ophiostomis rustica: Zikán \& Zikán 1944: 13.

Distribuição - Brasil (Goiás, Espírito Santo ao Rio Grande do

Sul), Paraguai, Argentina (Misiones), Uruguai.

4. Strangalia lyrata (Redtenbacher, 1867) (Figura 4)

Ophistomis lyrata Redtenbacher 1867: 190, Zajciw 1972: 48.

Ophiostomis lyrata: Zikán \& Zikán 1944: 13.

Ophistomis discophora: Zajciw 1972: 47.

Ophiostomis discophora: Zikán \& Zikán 1944: 12.

Distribuição - Brasil (Espírito Santo a São Paulo).

5. Strangalia melanura (Redtenbacher, 1867) (Figura 5)

Euryptera melanura Redtenbacher 1867: 189, Zikán \& Zikán

1944: 13, Zajciw 1972: 48.

Euryptera dimidiata: Zikán \& Zikán 1944: 13, Zajciw 1972: 48.

Distribuição - Brasil (Minas Gerais ao Rio Grande do Sul).

\section{SUBFAMÍLIA PARANDRINAE}

Tribo Parandrini

1. Acutandra murrayi (Lameere, 1912)

Parandra murrayi Lameere 1912: 115.

Acutandra murrayi: Santos-Silva 2002: 51.

Distribuição - Brasil (Minas Gerais ao Rio Grande do Sul).

2. Parandra (Hesperandra) expectata Lameere, 1902

(Figura 6)

Parandra expectata Lameere 1902: 69.

Parandra (Archandra) expectata: Zikán \& Zikán 1944: 2.

Parandra (Archandra) montana: Zajciw 1972: 47.

Distribuição - Brasil (Bahia ao Rio Grande do Sul), Argentina,

Paraguai e Uruguai.

3. Parandra (Parandra) brasilica Zikán, 1948 (Figura 7)

Parandra (Archandra) brasilica Zikán 1948: 31, Zajciw 1972:

46.

Parandra (Archandra)cribraria: Zikán 1948: 34.

Parandra (Archandra) melzeri: Zajciw 1972: 47.

Distribuição - Brasil (Minas Gerais a Santa Catarina).

4. Parandra (Parandra) glabra (Degeer, 1774)* (Figura 8)

Attelabus glaber Degeer 1774: 352.

Parandra (Archandra) glabra: Zikán \& Zikán 1944: 2, Zajciw

1972: 46.

Parandra (Archandra) lucida: Zajciw 1972: 46.

Distribuição - México a Argentina e Antilhas.

5. Parandra (Parandra) minuscula Zikán, 1948* (Figura 9)

Parandra (Archandra) minuscula Zikán 1948: 38, Zajciw

1972: 47.

Parandra (Archandra) costa-limai: Zajciw 1972: 46.

Parandra (Archandra) itatiayaensis: Zikán 1948: 39.

Distribuição - Brasil (Minas Gerais, Rio de Janeiro, São Paulo).

6. Parandra (Parandra) monnei (Santos-Silva, 2001)

Hesperandra monnei Santos-Silva 2001b: 229.

Distribuição - Brasil (Rio de Janeiro, São Paulo, Santa Catarina).

7. Parandra (Parandra) ubirajarai (Santos-Silva, 2001) (Figura 10)

Hesperandra ubirajarai Santos-Silva 2001a: 216.

Distribuição - Brasil (Minas Gerais a Santa Catarina).

8. Parandra (Tavandra) longicollis Thomson, 1861

(Figura 11)

Parandra longicollis Thomson, 1861b: 83.

Parandra (Archandra) longicollis: Zikán \& Zikán 1944: 2,

Zajciw 1972: 46.

Distribuição - Amplamente distribuída na América do Sul.

\section{SUBFAMÍLIA PRIONINAE}

Tribo Anacolini

1. Myzomorphus quadripunctatus (Gray, 1831)* (Figuras 12, 13)

Anacolus quadripunctatus Gray in Griffith \& Pidgeon 1831: pr. 70 .

Myzomorphus quadripunctatus: Galileo 1987b: 576.

Myzomorphus quadrimaculatus: Zikán \& Zikán 1944: 4, Zajciw 1972: 47.

Distribuição - Colômbia, Venezuela, Guiana Francesa, Peru, Brasil (Amazônia, Bahia a Santa Catarina).

Tribo Callipogonini

2. Callipogon (Orthomegas) jaspideum (Buquet, 1844)

(Figura 14) 

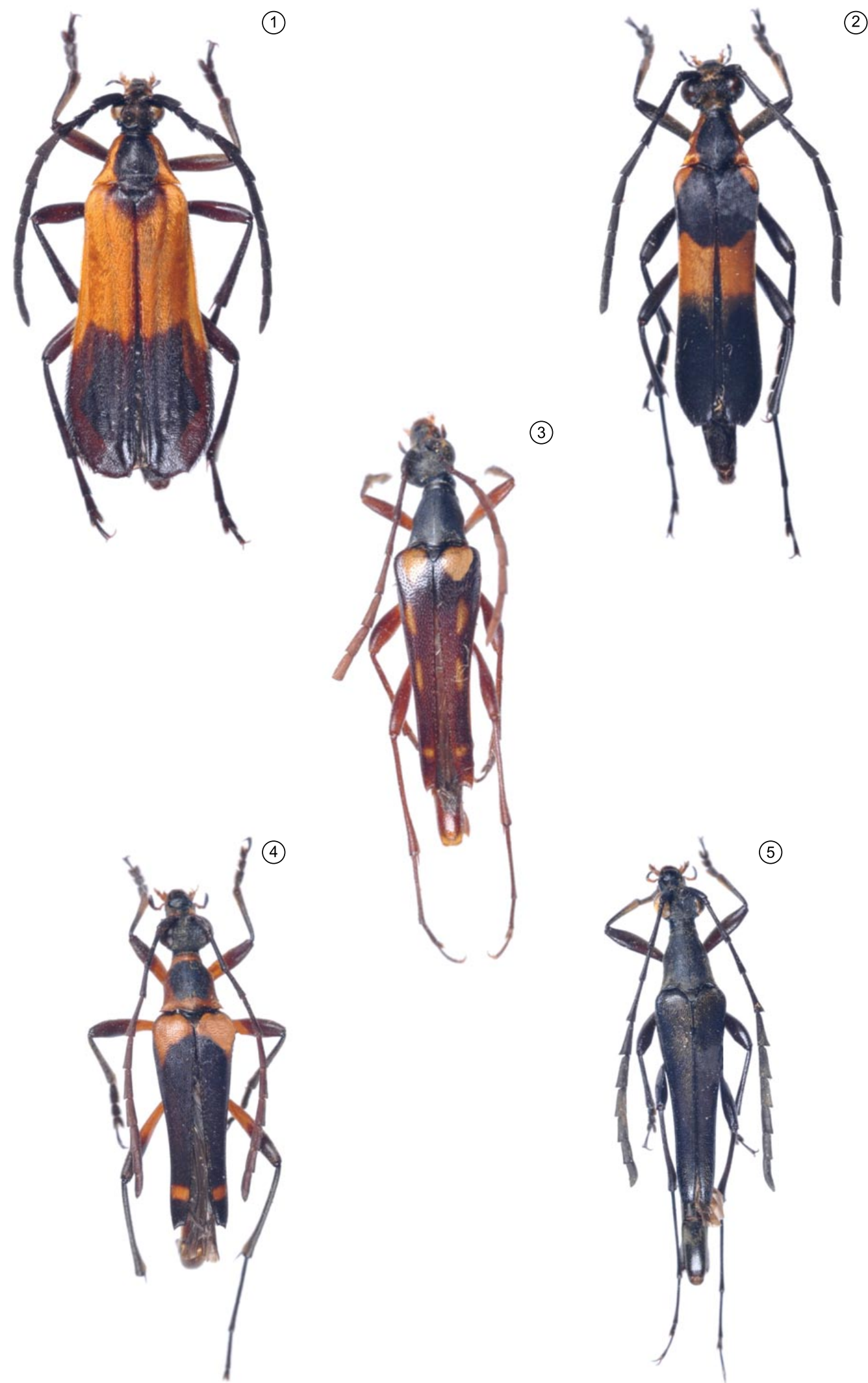

(3)

Figuras 1-5. 1) Euryptera unilineatocollis Fuchs, 1956; 2) Strangalia flavocincta (Thomson, 1861); 3) Strangalia fulvicornis (Bates, 1872); 4) Strangalia lyrata (Redtenbacher, 1867); 5) Strangalia melanura (Redtenbacher, 1867).

Figure 1-5. 1) Euryptera unilineatocollis Fuchs, 1956; 2) Strangalia flavocincta (Thomson, 1861); 3) Strangalia fulvicornis (Bates, 1872); 4) Strangalia lyrata (Redtenbacher, 1867); 5) Strangalia melanura (Redtenbacher, 1867). 

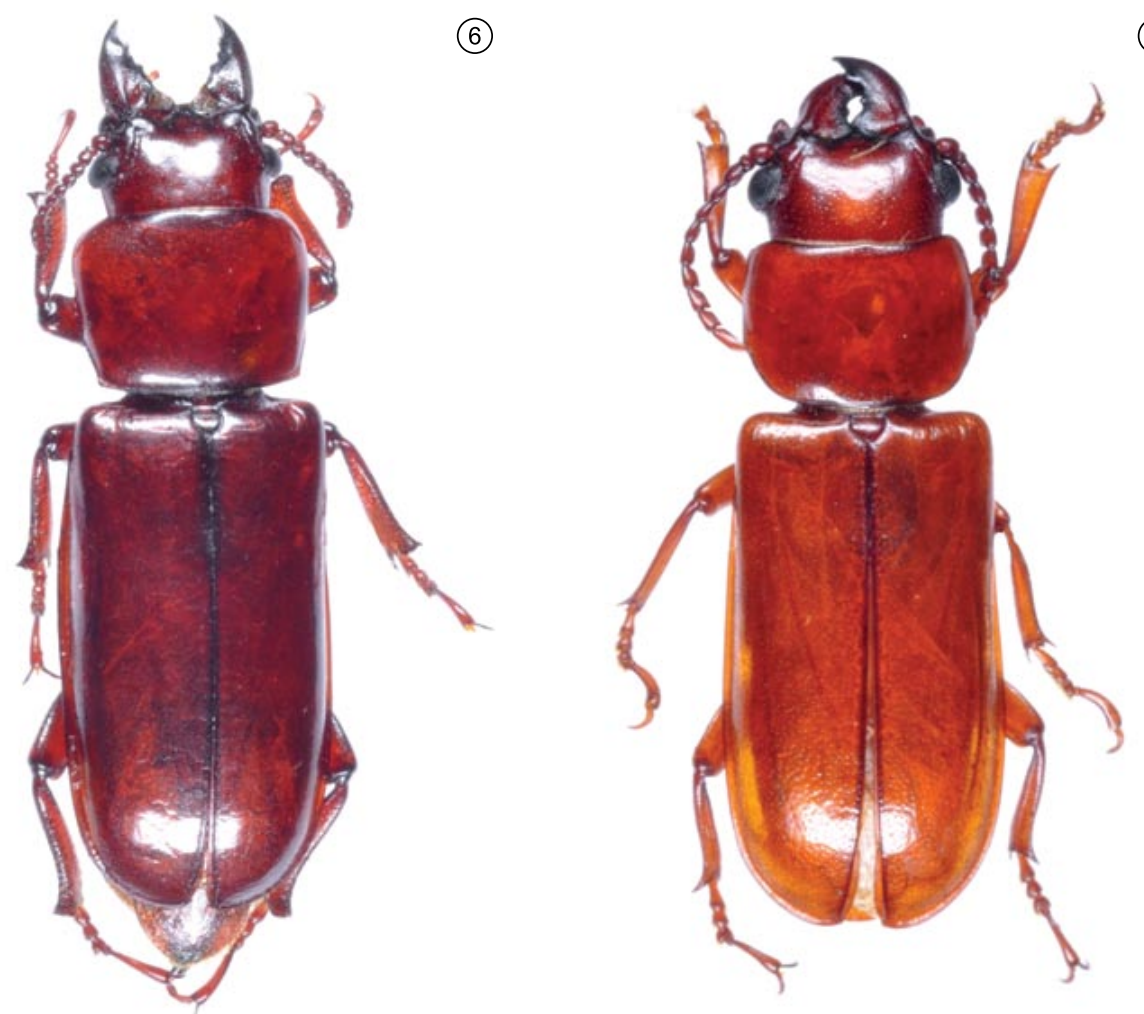

(7)

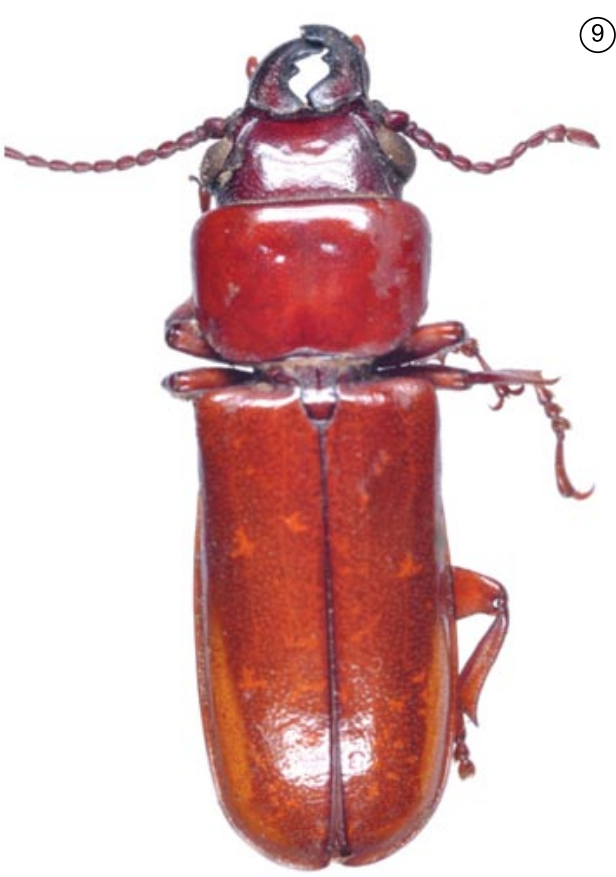

(9)

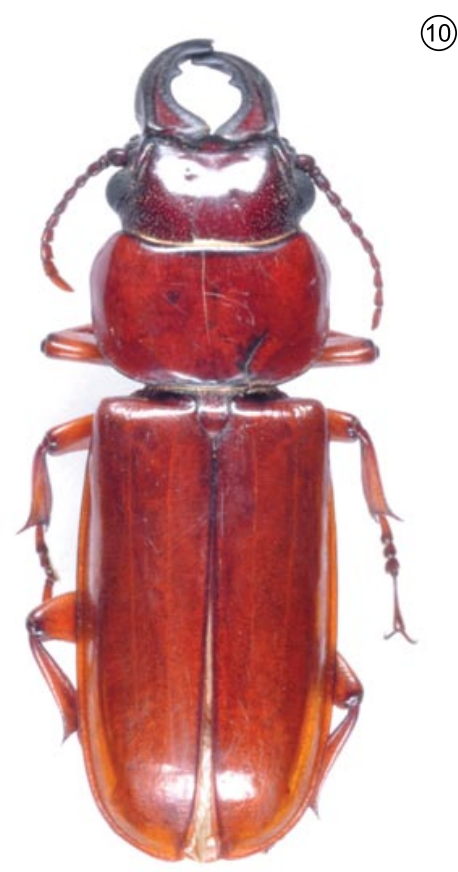

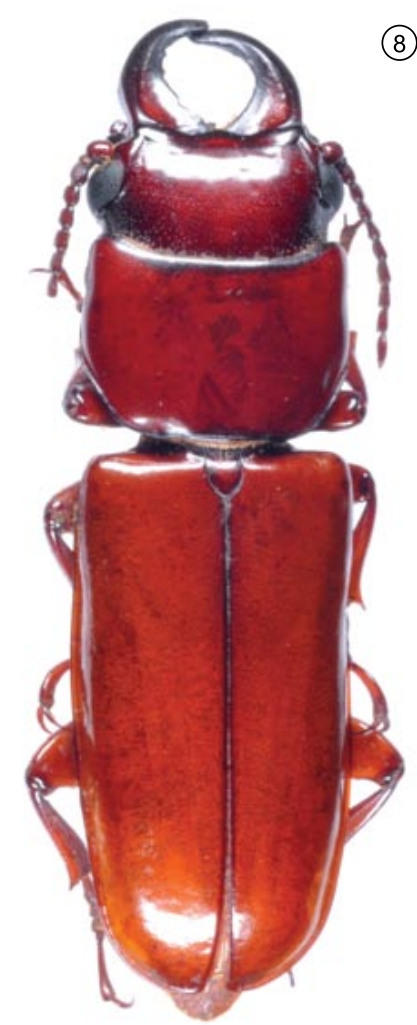

(8)

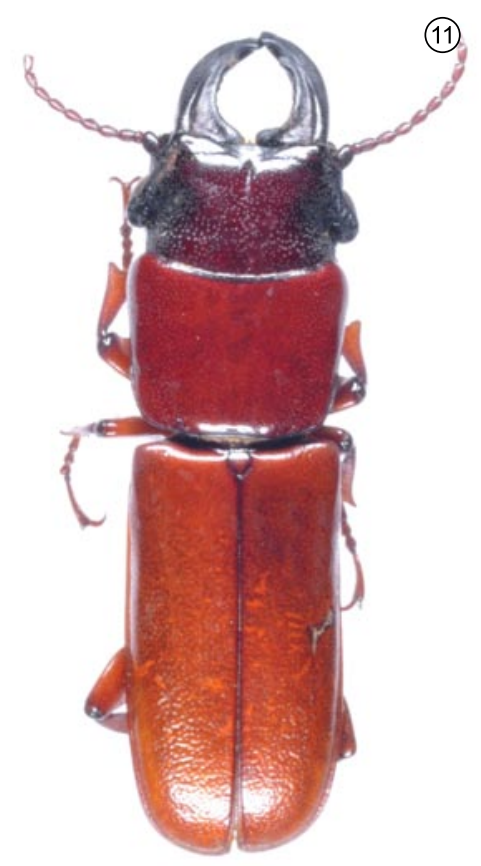

Figuras 6-11. 6, Parandra (Hesperandra) expectata Lameere, 1902; 7, Parandra (Parandra) brasilica Zikán, 1948; 8, Parandra (Parandra) glabra (Degeer, 1774); 9, Parandra (Parandra) minuscula Zikán, 1948; 10, Parandra (Parandra) ubirajarai (Santos-Silva, 2001); 11, Parandra (Tavandra) longicollis Thomson, 1861.

Figure 6-11. 6) Parandra (Hesperandra) expectata Lameere, 1902; 7) Parandra (Parandra) brasilica Zikán, 1948; 8) Parandra (Parandra) glabra (DeGeer, 1774); 9) Parandra (Parandra) minuscula Zikán, 1948; 10) Parandra (Parandra) ubirajarai (Santos-Silva, 2001); 11) Parandra (Tavandra) longicollis Thomson, 1861. 
Monné, M.L. et al.
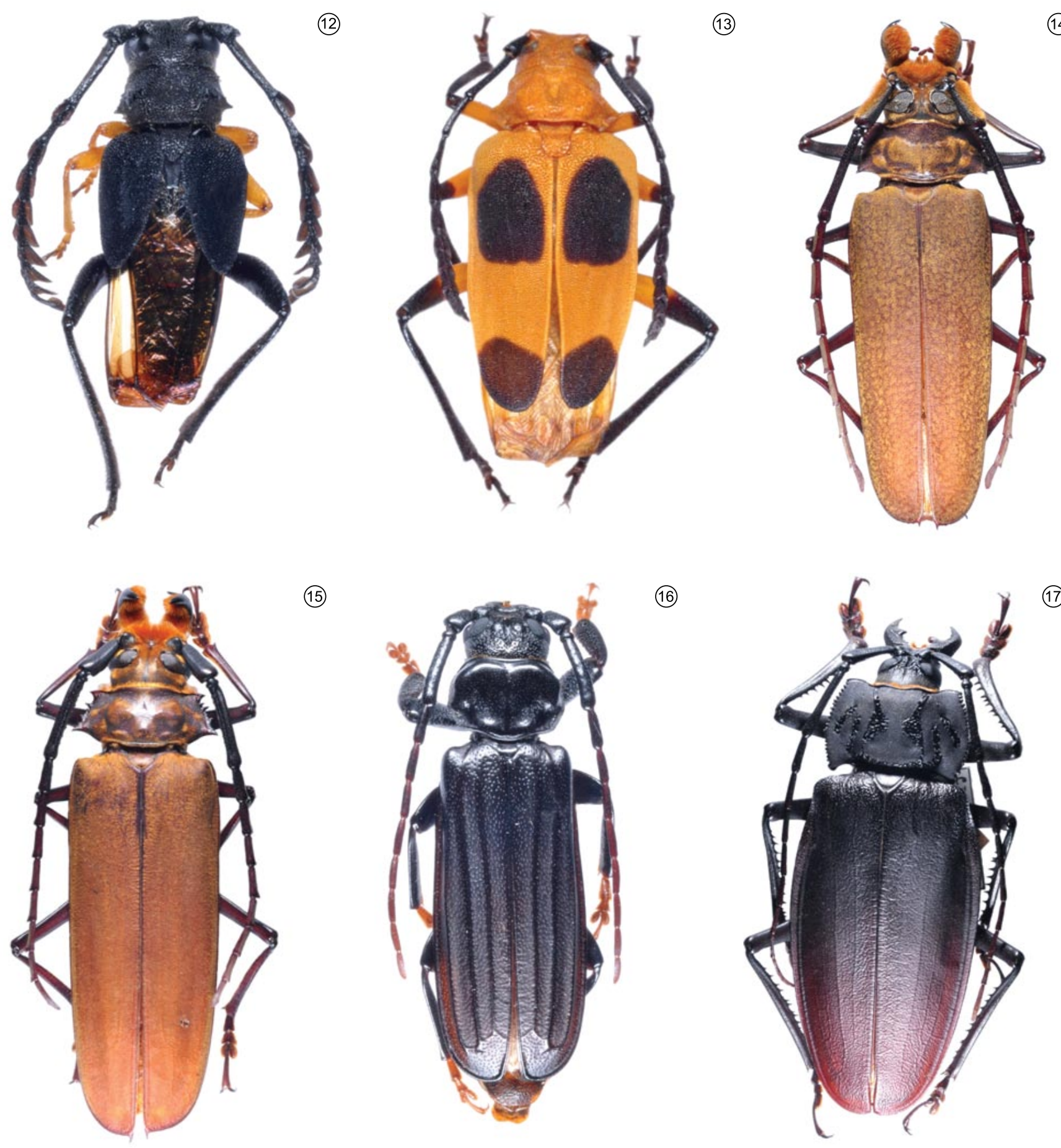

(15)

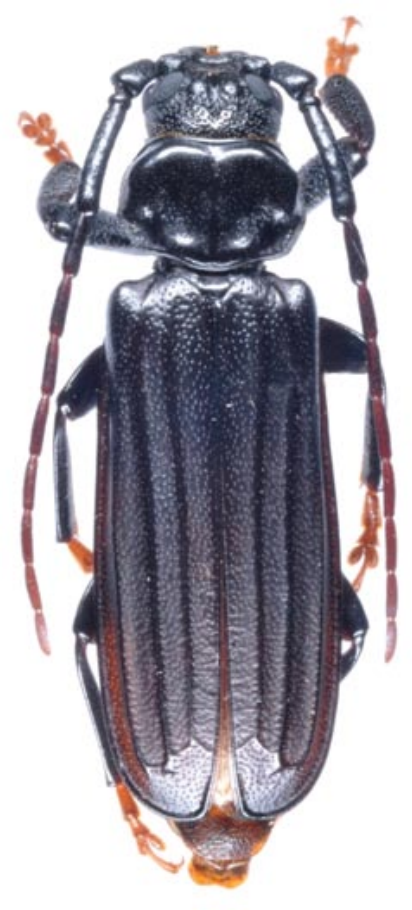

(16)

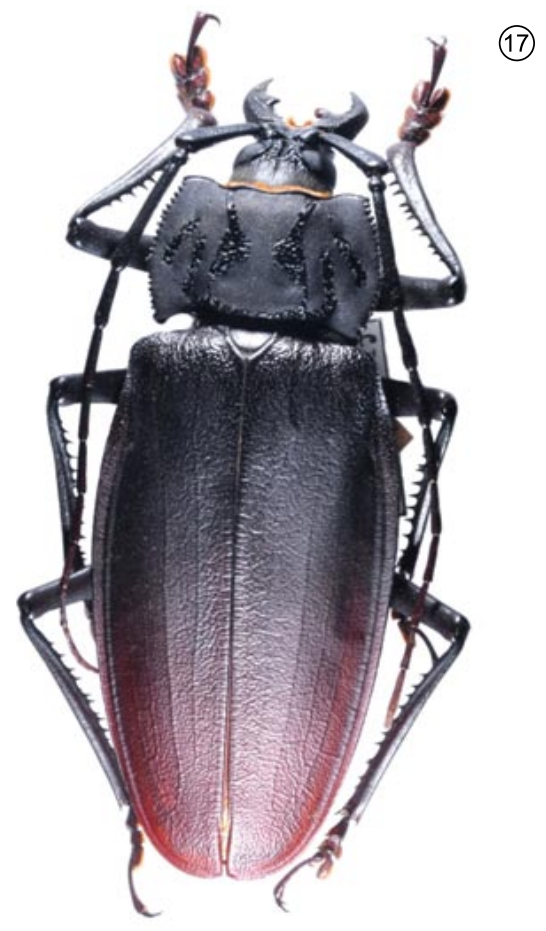

Figuras 12-17. Myzomorphus quadripunctatus (Gray, 1831), 12) macho, 13) fêmea; 14) Callipogon (Orthomegas) jaspideum (Buquet, 1844); 15) Callipogon (Orthomegas) similis (Gahan, 1894); 16) Chorenta reticulata (Dalman, 1817); 17) Ctenoscelis (Ctenoscelis) acanthopus (Germar, 1824).

Figura 12-17. Myzomorphus quadripunctatus (Gray, 1831), 12 male, 13 female; 14, Callipogon (Orthomegas) jaspideum (Buquet, 1844); 15) Callipogon (Orthomegas) similis (Gahan, 1894); 16) Chorenta reticulata (Dalman, 1817); 17) Ctenoscelis (Ctenoscelis) acanthopus (Germar, 1824). 
Lepturinae, Parandrinae e Prioninae do Parque Nacional do Itatiaia
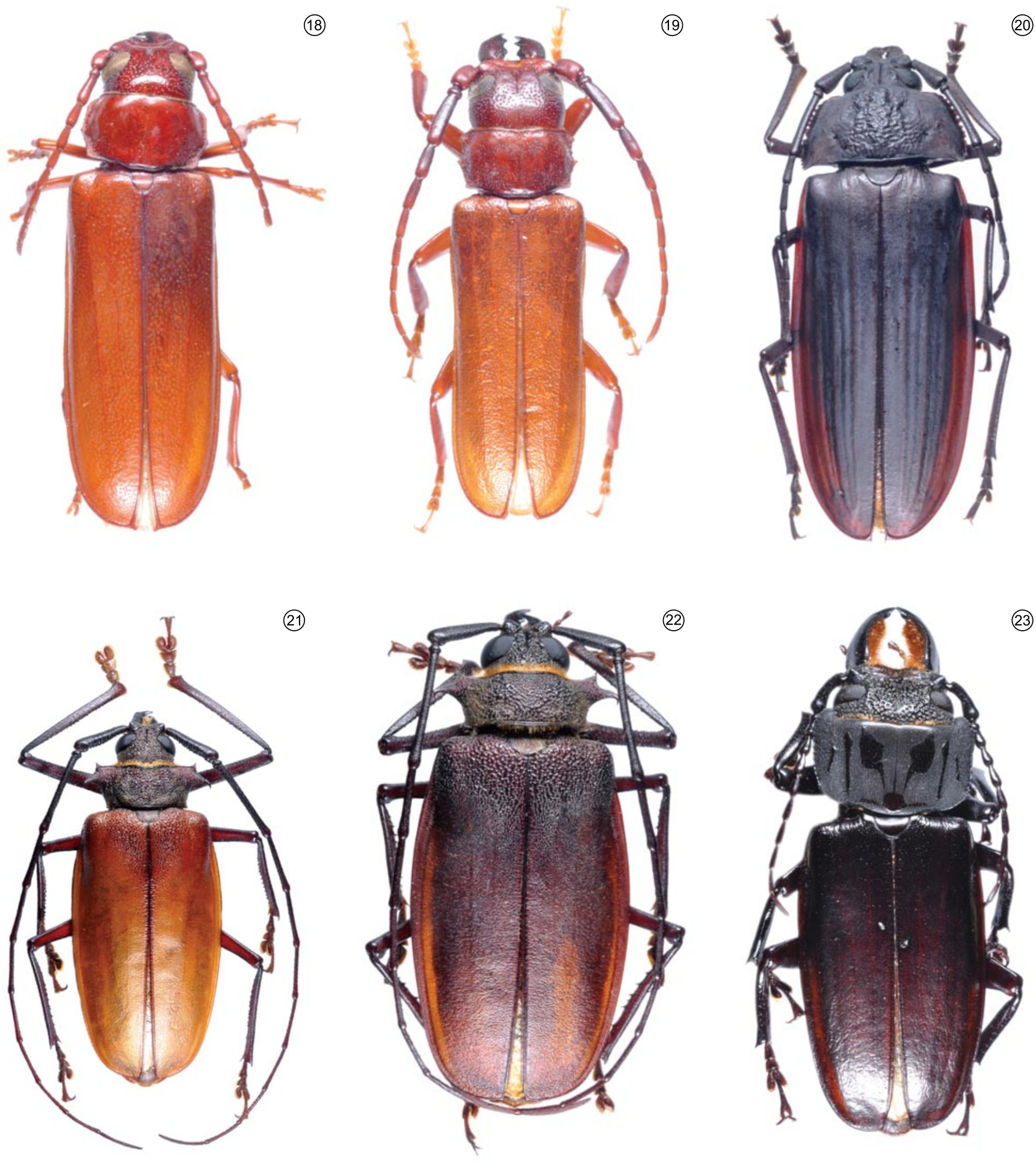

Figuras 18-23. 18) Hephialtes mourei Santos-Silva, 2004; 19) Hephialtes ruber (Thunberg, 1822); 20) Navosoma luctuosum (Schoenherr, 1817); 21) Ancistrotus aduncus Buquet, 1853; 22) Ancistrotus uncinatus (Klug, 1825); 23) Mallodon spinibarbis (Linnaeus, 1758).

Figure 18-23. 18) Hephialtes mourei Santos-Silva, 2004; 19) Hephialtes ruber (Thunberg, 1822); 20) Navosoma luctuosum (Schoenherr, 1817); 21) Ancistrotus aduncus Buquet, 1853; 22) Ancistrotus uncinatus (Klug, 1825); 23) Mallodon spinibarbis (Linnaeus, 1758). 

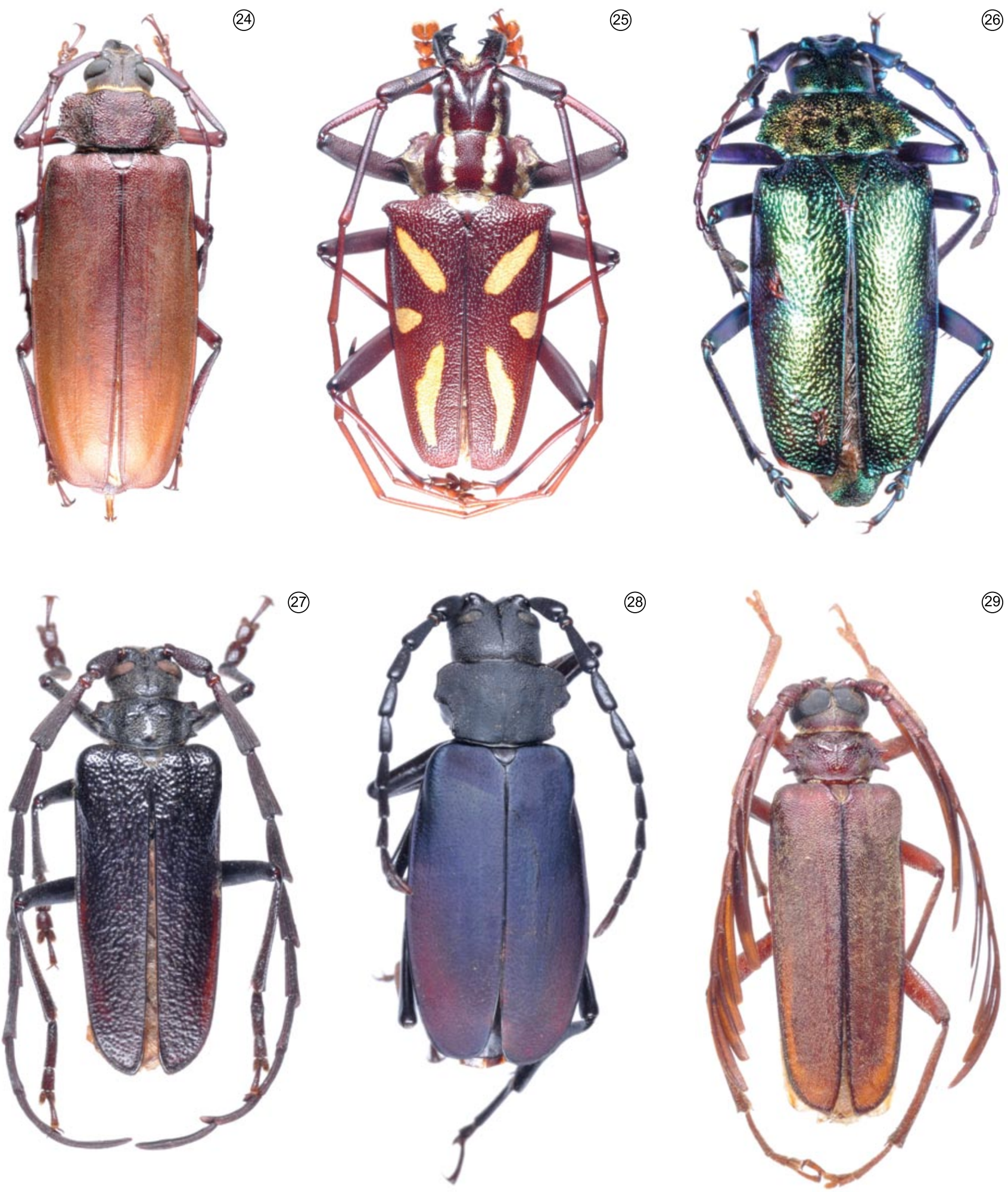

Figuras 24-29. 24) Mecosarthron buphagus Buquet, 1840; 25) Poecilopyrodes pictus (Perty, 1832); 26) Pyrodes nitidus (Fabricius, 1787); Meroscelisus apicalis White, 1853, 27) macho, 28) fêmea; 29) Polyoza lacordairei Audinet-Serville, 1832.

Figures 24-29. 24) Mecosarthron buphagus Buquet, 1840; 25) Poecilopyrodes pictus (Perty, 1832); 26) Pyrodes nitidus (Fabricius, 1787); Meroscelisus apicalis White, 1853, 27) male; 28) female; 29) Polyoza lacordairei Audinet-Serville, 1832. 

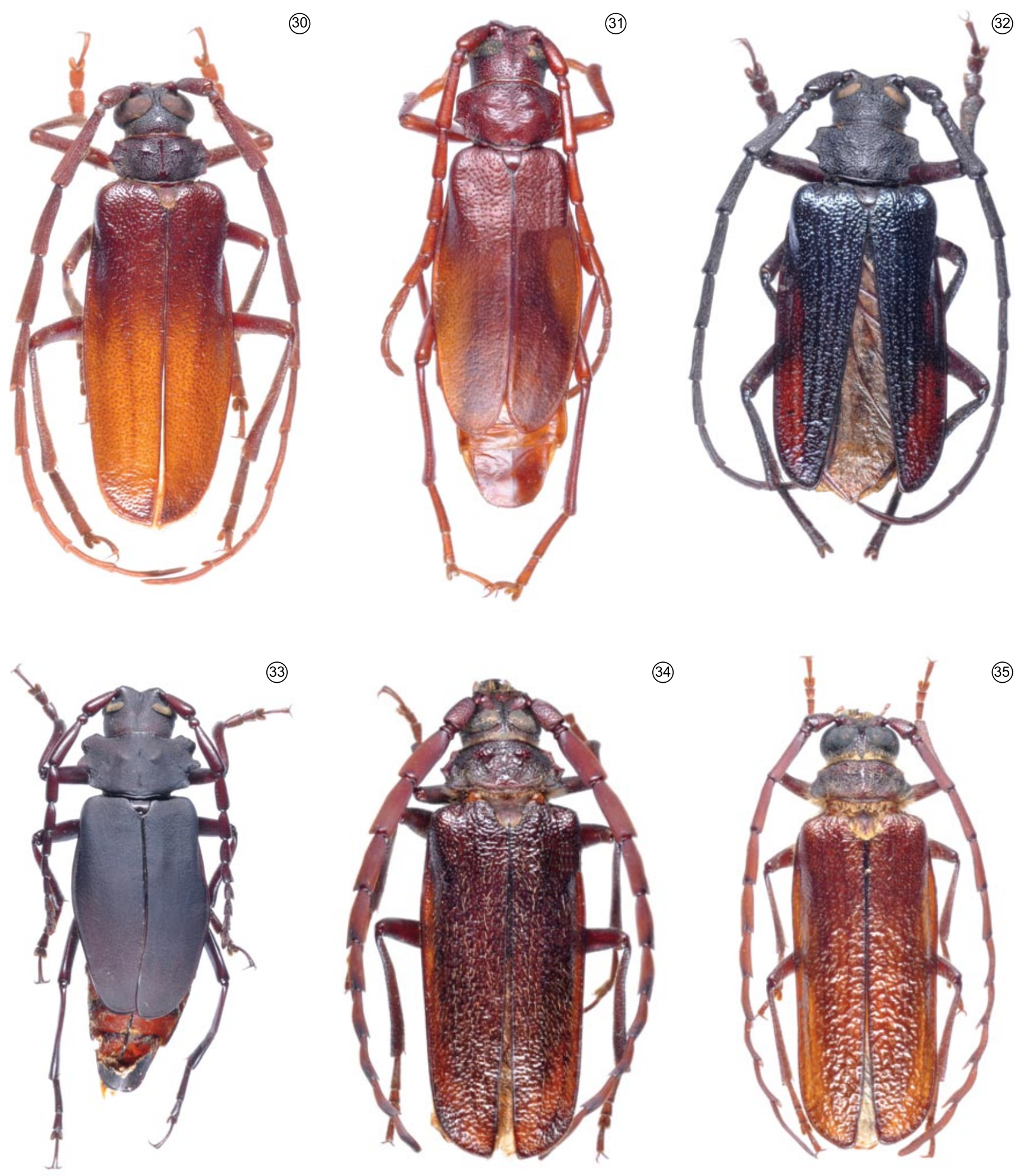

Figuras 30-35. Meroscelisus servillei Thomson, 1865, 30) macho, 31) fêmea; Meroscelisus violaceus Audinet-Serville, 1832, 32) macho, 33) fêmea; 34) Quercivir dohrni Lameere, 1912; 35) Quercivir gounellei Lameere, 1912.

Figures 30-35. Meroscelisus servillei Thomson, 1865, 30) male, 31) female; Meroscelisus violaceus Audinet-Serville, 1832, 32) male, 33) female; 34) Quercivir dohrni Lameere, 1912; 35) Quercivir gounellei Lameere, 1912. 
212.

Orthomegas jaspideus Buquet in Guérin-Méneville 1844:

Callipogon (Orthomegas) jaspideus: Zajciw 1972: 47.

Distribuição - Brasil (Pernambuco ao Rio Grande do Sul), Paraguai e Argentina.

3. Callipogon (Orthomegas) similis (Gahan, 1894) (Figura 15)

Orthomegas similis Gahan 1894: 223.

Callipogon (Orthomegas) similis: Zikán \& Zikán 1944: 3, Zajciw 1972: 47.

Distribuição - Brasil (Minas Gerais a Santa Catarina).

4. Chorenta reticulata (Dalman, 1817)* (Figura 16)

Prionus reticulatus Dalman in Schoenherr 1817: 147.

Stictosomus (Anacanthus) reticulates: Zikán \& Zikán 1944:

3, 45, Zajciw 1972: 47.

Distribuição - Brasil (Bahia ao Rio Grande do Sul), Paraguai e Argentina.

5. Ctenoscelis (Ctenoscelis) acanthopus (Germar, 1824) (Figura 17)

Prionus acanthopus Germar 1824: 467.

Ctenoscelis acanthopus: Zikán \& Zikán 1944: 3, 44.

Ctenoscelis (Ctenoscelis) acanthopus: Zajciw 1972: 47.

Distribuição - Brasil (Bahia ao Rio Grande do Sul), Paraguai e Argentina.

6. Hephialtes mourei Santos-Silva, 2004 (Figura 18)

Hephialtes mourei Santos-Silva 2004: 35.

Distribuição - Brasil (Minas Gerais, Rio de Janeiro ao Paraná).

7. Hephialtes ruber (Thunberg, 1822)* (Figura 19)

Trachyderes ruber Thunberg, 1822: 305.

Stictosomus (Anacanthus) rubber: Zikán \& Zikán 1944: 3,

Zajciw 1972: 47.

Distribuição - Guiana Francesa e Brasil.

8. Navosoma luctuosum (Schoenherr, 1817) (Figura 20)

Prionus luctuosus Schoenherr 1817: 346.

Callipogon (Navosoma) luctuosus: Zikán \& Zikán 1944: 3, Zajciw 1972: 47.

Distribuição - Brasil (Bahia ao Rio Grande do Sul, Mato Grosso), Paraguai, Uruguai e Argentina.

Tribo Macrodontiini

9. Ancistrotus aduncus Buquet, 1853* (Figura 21)

Ancistrotus aduncus Buquet 1853: xxiv, Bleuzen 1994: 37.

Distribuição - Brasil (Rio de Janeiro).

10. Ancistrotus uncinatus (Klug, 1825)* (Figura 22)

Prionus uncinatus Klug 1825: 454.

Ancistrotus uncinatus: Zikán \& Zikán 1944: 3.

Ancistrotus (Ancistrotus) uncinatus: Zajciw 1972: 47.

Distribuição - Brasil (Minas Gerais, Rio de Janeiro).

Tribo Macrotomini

11. Mallodon spinibarbis (Linnaeus, 1758)*(Figura 23)

Cerambyx spinibarbis Linnaeus 1758: 390.

Stenodontes (Mallodon) spinibarbis: Zikán \& Zikán 1944:

3.

Stenodontes (Orthomallodon) spinibarbis: Zajciw 1972: 47. Distribuição - México a Argentina.

12. Mecosarthron buphagus Buquet, 1840* (Figura 24)

Mecosarthron buphagus Buquet 1840: 172, Zikán \& Zikán

1944: 2, Zajciw 1972: 47.

Distribuição - Brasil (Bahia ao Paraná).
Tribo Mallaspini

13. Poecilopyrodes pictus (Perty, 1832) (Figura 25)

Prionus pictus Perty 1832: 85.

Pyrodes pictus: Zikán \& Zikán 1944: 3.

Pyrodes (Pyrodes) pictus: Zajciw 1972: 47.

Poecilopyrodes pictus: Galileo \& Martins 1992: 382.

Distribuição - Brasil (Bahia a São Paulo).

14. Pyrodes nitidus (Fabricius, 1787) (Figura 26)

Prionus nitidus Fabricius 1787: 128.

Pyrodes nitidus: Zikán \& Zikán 1944: 3.

Pyrodes (Pyrodes) nitidus: Zajciw 1972: 47.

Distribuição - Brasil (Alagoas ao Rio Grande do Sul, Mato

Grosso do Sul), Paraguai e Argentina (Misiones).

Tribo Meroscelisini

15. Meroscelisus apicalis White, 1853 (Figuras 27, 28)

Meroscelisus apicalis White 1853: 26.

Distribuição - Brasil (Espírito Santo a Santa Catarina).

Esta espécie está citada para o Rio de Janeiro no Estado do Rio de Janeiro, registra-se pela primeira vez para Itatiaia.

Material examinado. BRASIL, Rio de Janeiro: Itatiaia (Parque Nacional do Itatiaia), Maromba, 1.200m, 1 fêmea, 26.XII.1953, Seabra \& Alvarenga col. (MNRJ).

16. Meroscelisus servillei Thomson, 1865 (Figuras 30, 31)

Meroscelisus servillei Thomson 1865: 577, Galileo 1987a: 243.

Distribuição - Brasil (Espírito Santo ao Rio Grande do Sul), Paraguai e Argentina.

17. Meroscelisus violaceus Audinet-Serville, 1832 (Figuras 32, 33)

Meroscelisus violaceus Audinet-Serville 1832: 158, Zikán \& Zikán 1944: 4, Zajciw 1972: 47.

Distribuição - Brasil (Bahia a Santa Catarina) e Paraguai.

18. Polyoza lacordairei Audinet-Serville, 1832 (Figura 29)

Polyoza lacordairei Audinet-Serville 1832: 167, Zikán \& Zikán 1944: 4, Zajciw 1972: 47.

Distribuição - Brasil (Goiás, Bahia ao Rio Grande do Sul), Paraguai, Argentina e Uruguai.

19. Quercivir dohrni Lameere, 1912 (Figura 34)

Quercivir dohrni Lameere 1912b: 37.

Distribuição - Brasil (Rio de Janeiro a Santa Catarina).

Esta espécie está citada para o Rio de Janeiro no Estado do Rio de Janeiro, registra-se pela primeira vez para Itatiaia.

Material examinado. BRASIL, Rio de Janeiro: Itatiaia (Parque Nacional do Itatiaia), 1 macho, 1950 (MNRJ).

20. Quercivir gounellei Lameere, 1912 (Figura 35)

Quercivir gounellei Lameere 1912b: 38, Galileo 1987a: 219.

Quercivir zikani: Zikán \& Zikán 1944: 4, Zajciw 1972: 47.

Distribuição - Brasil (Minas Gerais ao Rio Grande do Sul).

\section{Agradecimentos}

Ao IBAMA pela autorização de coleta No. 16928-1. Ao Diretor de Pesquisa do PNI, Leo Nascimento, por incentivar a realização dos estudos no parque. Ao Joel, funcionário do parque, pelas gentilezas durante as estadias na Casa do Pesquisador no PNI. 


\section{Referências Bibliográficas}

AUDINET-SERVILLE, J.G. 1832. Nouvelle classification de la famille des longicornes. Ann. Soc. Ent. de Fr. 1(1):118-201.

BATES, H.W. 1872. On the longicorn Coleoptera of Chontales, Nicaragua. Trans. Ent. Soc. London 1872:163-238.

BLEUZEN, P. 1994. Les Coléoptères du Monde. Prioninae I. Sci. Nat. 21:1-92.

BUQUET, J.B.L. 1840. Coléoptères nouveaux. Revue Zool. 1840:172-173.

BUQUET, J.B.L. 1884. In Iconographie du règne animal de G. Cuvier, ou réprésentation d'après nature de l'une des espèces les plus remarquables et souvent non figurées de chaque genre d'animaux. Avec un texte descriptif mis au courant de la science.Ouvrage pouvant servir d'atlas a tous les traités de zoologie. (F.E. Guérin-Méneville). Insectes 7:576.

BUQUET, J.B.L. 1853. Erratum. Bull. Soc. Ent. Fr. 1853:24.

DALMAN, J.W. 1817. Appendix. In Synonymia insectorum, oder: Versuch einer synonymie aller bisher bekannten Insectten; nach Fabricii Systema Eleutheratorum \& c. geordnet. Eleutherata oder Käfer (C.J. Schoenherr). Skara, Lewerentzischen Buchdruekerey, v.1, n.3, 506p.

DEGEER, C. 1774. Mémoires pour servir à l'histoire des insectes. Pierre Hesselberg, Stockholm. v.4, 455p

FABRICIUS, J.C. 1787. Mantissa insectorum sistens eorum species nuper detectas adiectis characteribus genericis, differentiis specificis, emendationibus, observationibus. Proft, Hafniae, 348p.

FUCHS, E. 1956. 2. Beitrag zur Kenntnis der neotropischen Cerambyciden (Col.). Ent. Arb. Mus. Frey 7(2):567-576.

GAHAN, C.J. 1894. Descriptions of some new species of Prionidae. Ann. Mag. Nat. Hist. (6)14:221-227.

GALILEO, M.H.M. 1987a. Sistemática das tribos Meroscelisini e Anacolini (Coleoptera, Cerambycidae, Prioninae) nas Américas. I. Meroscelisini. Rev. Bras. Ent. 31(2):141-267.

GALILEO, M.H.M. 1987b. Sistemática das tribos Meroscelisini e Anacolini (Coleoptera, Cerambycidae, Prioninae) nas Américas. II. Anacolini. Rev. Bras. Ent. 31(4):481-705.

GALILEO, M.H.M. \& MARTINS, U.R. 1992. Revisão da tribo Mallaspini Thomson, 1860 (Coleoptera, Cerambycidae, Prioninae). Rev. Bras. Ent. 36(2):293-422.

GERMAR, E.F. 1824. Insectorum species novae aut minus cognitae, descriptionibus illustratae. Hendel \& Sons, Halae, 624p.

GRAY, G. 1831. In Griffith, E. \& Pidgeon, E. The animal kingdom arranged in conformity with its organization by the Baron Cuvier, with supplementary addition to each order. Insects, London, 796p.

Brasil. Ministério do Meio Ambiente. Instituto Brasileiro do Meio Ambiente e dos Recursos Naturais Renováveis - IBAMA. <http://www.icmbio.gov. br/parna_itatiaia/> (último acesso em 10/03/2010).

KLUG, J.C. 1825. Entomologiae Brasilianae specimen sistens insectorum coleopterorum nondum descriptorum centuriam. Nova Acta Acad. Caesar. Leopold. Carol. 12(2):421-476.

LAMEERE, A.A. 1902. Révision des prionides (Premier mémoire Parandrides). Ann. Soc. Ent. Belg. 46:59-111.

LAMEERE, A.A. 1912. Révision des prionides (Vingt-et-unième mémoire Anacolines). Mém. Soc. Ent. Belg. 21:1-188.

LINNAEUS, C. 1758. Systema naturae per regne tria naturae secundum classes, ordines, genera, species, cum characteribus, differentiis, synonymis, locis. Holmiae, 823p.

MARTINS, U.R. 1997. Cerambycidae Sul-Americanos (Coleoptera): taxonomia. Sociedade Brasileira de Entomologia, São Paulo.

MONNÉ, M.A. 2005a. Catalogue of the Cerambycidae (Coleoptera) of the Neotropical Region. Part I. Subfamily Cerambycinae. Zootaxa 946:1765.

MONNÉ, M.A. 2005b. Catalogue of the Cerambycidae (Coleoptera) of the Neotropical Region. Part II. Subfamily Lamiinae. Zootaxa 1023:1-760.

MONNÉ, M.A. 2006. Catalogue of the Cerambycidae (Coleoptera) of the Neotropical Region. Part III. Subfamilies Parandrinae, Prioninae,
Anoplodermatinae, Aseminae, Spondylidinae, Lepturinae, Oxypeltinae, and addenda to the Cerambycinae and Lamiinae. Zootaxa 1212:1-244.

MONNÉ, M.L. \& MONNÉ, M.A. 2008. The tribe Lepturini in South America (Coleoptera: Cerambycidae: Lepturinae). Zootaxa 1858:37-52.

MONNÉ, M.L., MONNÉ, M.A. \& MERMUDES, J.R.M. 2009. Inventário das espécies de Cerambycinae (Insecta, Coleoptera, Cerambycidae) do Parque Nacional do Itatiaia, RJ, Brasil. Biota Neotr. 9(3):1-30.

PERTY, J.A.M. 1832. De insectorum in America meridionali habitantiam vitae genere, moribus ac distributione geographica observationes nonnullae. In Delectus animalium articulatorum quae in itinere per Brasilian annis MDCCCXVII-MDCCCXX jussu at auspicis Maximiliani Josephi I. Bavariae regis augustissime peracto collegerunt Dr. J. B. de Spix et Dr. C. F. Ph. de Martius. Monachii, p.61-124.

REDTENBACHER, L. 1867. Coleopteren. In Reise des österreichischen Fregatte Novara und die Erde in den Jahren 1857, 1858, 1859, unter der befehlen des Commodore B. von Wüllerstorf-Urbair. Zoologischer Theil, Wien, 249p.

SANTOS-SILVA, A. 2001a. Parandrinae neotropicais (Coleoptera, Cerambycidae) I: redescrição de Parandra degeeri Thomson, 1867 e descrição de uma nova espécie. Rev. Bras. Ent. 45(3):215-219.

SANTOS-SILVA, A. 2001b. Parandrinae neotropicais (Coleoptera, Cerambycidae) II: espécies de Hesperandra Arigony, 1977 com submento elevado e marginação lateral do protórax dos machos incompleta. Rev. Bras. Ent. 45(3):221-241.

SANTOS-SILVA, A. 2002. Notas e descrições em Parandrini (Coleoptera, Cerambycidae, Parandrinae). Iheringia Serie Zool. 92(2):29-52.

SANTOS-SILVA, A. 2004. Descrição de Hephialtes mourei sp. nov. e notas em Hephialtes Thomson e Braderochus mundus (White) (Coleoptera, Cerambycidae, Prioninae). Rev. Bras. Ent. 48(1):35-38.

SANTOS-SILVA, A. \& MARTINS, U.R. 2010. Subfamília Parandrinae. In Cerambycidae Sul-americanos (Coleoptera) taxonomia (U.R. Martins, org.). Sociedade Brasileira de Entomologia, São Paulo, p.5-79.

SCHOENHERR, C.J. 1817. Synonymia Insectorum, oder: Versuch einer Synonymia aller bisher bekannten Insecten; nach Fabricii Systema Eleutheratorum \&c. geordnet. Lewerentzischen Buchdruekerey 1(3):1-506

THOMSON, J. 1861a. Essai d'une classification de la famille des cérambycides et matériaux pour servir a une monographie de cette famille. BouchardHuzard, Paris, p.129-396.

THOMSON, J. 1861b. Monographie de la famille des parandrides. Musée Scientifique 2:73-87.

THOMSON, J. 1865. Diagnoses d'espèces nouvelles qui seront décrites dans l'appendix du systema cerambycidarum. Mém. Soc. Roy. Sci. Liège 19:353-578.

THUNBERG, C.P. 1822. Trachyderes insecti genus ulterius examinatum et auctum sex novis speciebus. Mém. Acad. Imp. Sci. de St.-Petersb. 1822:303-307.

WHITE, A. 1853. Catalogue of the coleopterous insects in the collection of the British Museum. Longicornia 1. British Museum, London, 174p.

ZAJCIW, D. 1972. Contribuição para o estudo da fauna dos longicórneos do Parque Nacional do Itatiaia (Coleoptera, Cerambycidae). Bras. Florest. 3:40-72.

ZIKÁN, J.F. 1948. O gênero Parandra Latr., 1804, com a descrição de 14 espécies novas (Col., Cerambycidae). Rev. Soc. Ent. Arg. 14:22-50

ZIKÁN, J.F. \& ZIKÁN, W. 1944. A inseto-fauna do Itatiaia e da Mantiqueira. Bolm. Min. Agric. 33(8):1-50. 